(Aus dem physiol. Institute der thierärztl. Hochschule in Budapest. Vorstand: Prof. Dr. F. Tangl.)

\title{
Zur Kenntniss des Chorionins und des Chorioningehaltes der Seidenspinnereier.
}

\author{
Von \\ Dr. K. Farkas, II. Assistent am Institut.
}

In vorstehender Arbeit (S. 490) wies ich darauf hin, dass zwischen dem Hühnerei und Seidenspinnerei bezüglich der Eischale bedeutende Unterschiede sind. Letztere enthält viel mehr und ganz andere organische Stoffe mit viel grösserem Gehalt an chemischer Energie. Auch ist die Schale vom Eiinhalt, im Gegensatz zum Hühnerei, vollständig kaum zu trennen. Eben desshalb wollte ich ursprünglich der Frage näher treten, ob die Eischale am Stoffwechsel im Ei theilnimmt oder nicht. Wenn ich auch das nicht entscheiden konnte, so haben meine Untersuchungen über die Schale des Seidenspinnereies einige mittheilenswerthe Daten geliefert, die ich im Folgenden anführe.

Bei meinen diesbezüglichen Untersuchungen hielt ich mich an die Versuche von Tichomiroff ${ }^{1}$ ), der die Schale des Seidenspinnereies am eingehendsten geprüft und bewiesen hat, dass sie nicht aus Chitin, sondern aus einer dem Chitin äusserlich ähnlichen Substanz besteht, die er "Chorionin“ nannte. Wesentliche Unterschiede bestehen in ibrer elementaren Zusammensetzung und Löslichkeit. Das Chorionin löst sich in verhältnissmässig schwachen Laugen vollkommen, während das Chitin auch in concentrirten Laugen unlöslich ist. Tichomiroff hat auch eine Methode zur Darstellung und quantitativen Bestimmung des Chorionins angegeben, die auch ich angewendet habe.

1) Tichomir off, Chem. Studien über die Entwicklung der Insecteneier. Zeitschr. f. physiol. Chemie Bd. 9 S. 518-532 und 566-567. 
Ich verwendete unbebrütete Eier meiner II. Versuchsreihe (siehe voranstehende Arbeit S. 493), die ich nach Tichomiroff auf folgende Weise verarbeitete:

Ungefähr $1 \mathrm{~g}$ Eier wurden in $1 \%$ iger Salzsäure fein zerrieben

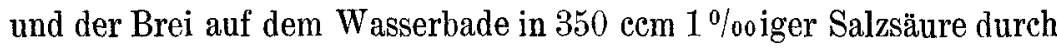
2 Stunden gekocht. Nach dem Abfiltriren der Salzsäure wurde die Masse noch 20 Minuten in ca. $300 \mathrm{ccm}$ destillirten Wassers gekocht und dann durch 36 Stunden in 300 cem Pepsinsalzsäure (1\% Pepsinum germanicum in $2 \%$ ige Salzsăure) bei Körpertemperatur künstlicher Verdauung ausgesetzt. Nachher wurde abermals in $1 \%$ iger Salzsäure 10 Minuten lang, dann in 3-4 Mal gewechseltem Alkohol, Aether gekocht, zuletzt mit Alkohol āā Aether ausgewaschen. Das zurückbleibende Chorionin wurde auf ein abgewogenes Filter gebracht und nach dem Trocknen - im Vacuum bei $85^{\circ} \mathrm{C}$. - gewogen.

Diese Methode weicht von der Tichomiroff'schen nur darin ab, dass ich nicht natürlichen, sondern künstlichen Magensaft benutzte.

Auf diese Art gewann ich aus den Eiern:

in der 1. Probe . . 10,46\%

" 2 . ". . . 10,60\% Chorionin.

Tichomiroff fand $8,87 \%$; meine Werthe sind also etwas höher. Bei der Wiederholung der Bestimmung, wobei ich nach Hoppe-Seyler bereiteten natürlichen Magensaft benutzte, bekam ich dasselbe Resultat:

$$
\begin{aligned}
& \text { in der 3. Probe . . . 10,40\% } \\
& \text { " } \quad 4 \text {. . . . 10,40\%. }
\end{aligned}
$$

In dem auf diese Weise erhaltenen Chorionin bestimmte ich den C-, N- und Energiegehalt. Nach mehreren gut übereinstimmenden Analysen ist der

$$
\begin{aligned}
& \text { C-Gehalt . . . . . . . . 49,63\% } \\
& \text { N-Gehalt . . . . . . . . } 15,64 \% \\
& \text { Energiehalt von } 1 \mathrm{~g} \text { Chorionin . } 5115 \text { cal. }{ }^{1} \text { ). }
\end{aligned}
$$

Die entsprechenden Werthe Tichomir off's weichen - wie es aus der folgenden Zusammenstellung ersichtlich ist - von den meinigen ab. In die Tabelle habe ich auch die Angaben Verson's ${ }^{2}$ ) aufgenommen, der die Eischalen für Keratin hielt. Seine Werthe

1) Die Verbrennung in der Berthelot'schen Bombe geschah in derselben Weise wie bei den leeren Eischalen; siehe meine voranstehende Arbeit S. 494.

2) Citirt nach Tichomiroff S. 566. 
betrachtete aber Tichomir off nicht für einwandsfrei, da die Eischalen nicht entsprechend gereinigt waren.

$$
\begin{array}{ccc}
\begin{array}{c}
\text { Nach } \\
\text { Tichomiroff }
\end{array} & \begin{array}{c}
\text { nach } \\
\text { Verson, }
\end{array} & \begin{array}{c}
\text { nach meinen } \\
\text { Versuchen }
\end{array} \\
\text { Eier } 8,87 \% & - & 10,46 \% \\
25,97 \% & - & 29,03 \%
\end{array}
$$

Chorioningehalt der unbebrüteten Eier $8,87 \% \quad-\quad 10,46 \%$

Das Chorionin enthält:

$$
\begin{aligned}
& \mathrm{C} \quad \cdot \quad 47,27 \% \quad 50,90 \% \quad 49,63 \% \\
& \mathrm{H} . \quad . \quad 6,71 \% \quad 7,11 \% \quad- \\
& \mathrm{N} \text {. . . } 16,93 \% \quad 17,20 \% \quad 15,64 \% \\
& \text { O . . 24,72\% } 19,33 \% \quad- \\
& \mathrm{S} . \cdot 3,67 \% \quad 4,38 \% \quad- \\
& \text { Asche . . } 0,70 \% \quad 1,09 \% \quad \text { - }
\end{aligned}
$$

Möglicher Weise sprechen die aus dieser Zusammenstellung ersichtlichen Differenzen der einzelnen Untersuchungen dafür, dass das Chorionin individuell verschieden, oder, dass es kein einheitlicher Körper ist.

Was den Energiegehalt (5115 cal.) des Chorionins betrifft, so steht dieser einer anderen Substanz des Seidenspinners, nämlich dem Fibroïn (4980 cal.) nahe, entfernt sich aber beträchtlich von dem des Chitins $\left(4650\right.$ cal.) wie auch des Keratins $\left.(\text { Wolle }=5510 \text { cal. })^{1}\right)$.

Aus der Menge und Zusammensetzung des Chorionins geht hervor, dass es einen grossen Theil des Stoff- und Energiegehaltes der unbebrüteten Eier bildet, und zwar fallen:

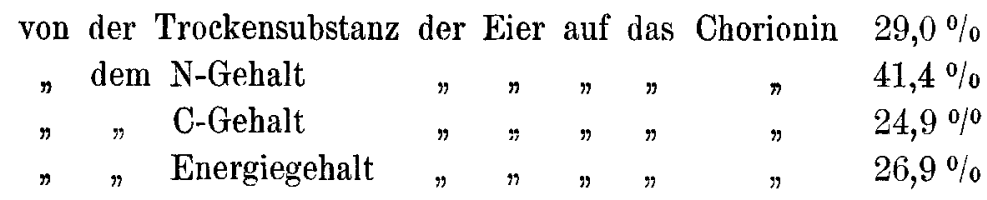

In den 10,46 g Chorionin von $100 \mathrm{~g}$ Eier sind enthalten:

$$
\begin{aligned}
& \text { Asche. . . } 0,07 \mathrm{~g} \text { (nach Tichomiroff) } \\
& \mathrm{N} \cdot \text {. . } 1,64 \text {, } \\
& \mathrm{C} \text {. . . } 5,71 \text {, } \\
& \text { Energie . . 53,5 Cal. }
\end{aligned}
$$

Vorausgesetzt, dass die Eischale zum allergrössten Theil aus Chorionin besteht, kann man nun die Zusammensetzung des Eiinhaltes durch einfache Subtraction aus den für das Gesammtei gefundenen

1) Börnstein-Landolt, Physik. Tabellen, 2. Aufl. 
550 K. Farkas: Zur Kenntn. des Chorionins u. des Chorioningehaltes etc.

Werthen (s. S. 504 der vorangehenden Arbeit) leicht berechnen. Demnach fanden sich in $100 \mathrm{~g}$ Eiern:

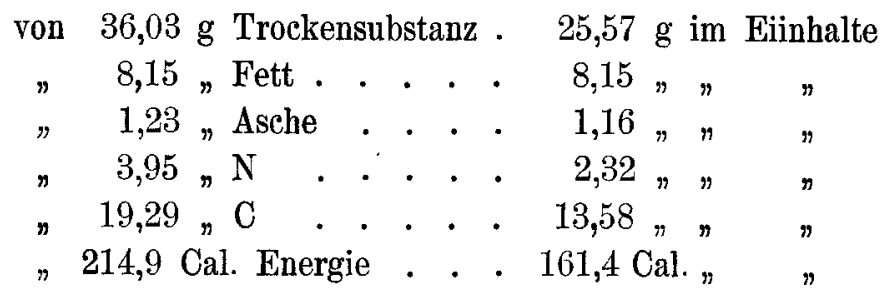

Zum Schlusse sei mir nur noch eine Bemerkung über die Bedeutung der Eischale gestattet. Sie kann entweder nur eine an der embryonalen Entwicklung unbetheiligte Schutzvorrichtung sein, oder aber sie könnte sich auch eventuell am Stoffwechsel betheiligen Ob Letzteres der Fall ist, konnte ich nicht entscheiden, da ich ebenso wie Tichomiroff den Chorioningehalt der Eier nur vor - und nicht nach - dem Bebrüten bestimmt habe. Es ist aber erwähnenswerth, dass die ausschlüpfenden Raupen, während ihres Auskriechens, nach $\mathrm{Haberlandt^{1 }}$ ) einen Theil der Eischalen, weleher durch ein alkalisches Secret der Raupen aufgeweicht wird, durchnagen und die abgenagten Theile schlucken. Es scheint nicht ausgeschlossen zu sein, dass die abgenagten Eischale-Partikelchen in dem alkalisehen Magensaft verdaut werden und auf diese Art der neugeborenen Raupe als Nahrung dienen können.

1) Fr. Haberlandt, Der Seidenspinner. Wien 1871. 